\title{
The Research of Microstructure UV Curing Mold Injection
}

\author{
Jian-yun $\mathrm{He}^{1,2}$, Xia Tang ${ }^{1,2}$, Le Chang ${ }^{1,2}$, Yu-mei Ding ${ }^{1,2}$, Wei-min Yang ${ }^{1,}$ \\ ${ }^{2}$, Peng-cheng $\mathrm{Xie}^{1,2}$, Xian-chun $\mathrm{Ye}^{1,2 *}$
}

\author{
1.Beijing University of Chemical Technology, Beijing 100029, China; 2.BUCT Xiamen Biological \\ Industry Research Institute co., LTD, Beijing 100029, China \\ jyhe2009@163.com China \\ 18612948131@163.com China
}

Keywords: Microstructure component; UV curing mold injection; Mold precision; Molding process; Replication accuracy; Guidelines

\begin{abstract}
Different from the traditional "physical manufacturing" mold injection, UV curing mold injection is a new manufacturing method which combines mold injection process and UV curing technology. On the basis of the traditional mold injection process the photochemical mold manufacturing process parameters had been studied, the effect of UV material temperature and the holding pressure on products precision had been analyzed and the quality of products microstructure morphology had been evaluated with cross section graph comparison method. Meanwhile, the micro-structure formability and replication accuracy of triangular edge cross section of miniature products had been researched. The experimental results shows that it is possible for UV curing mold injection to replace the thermoplastic mold injection. UV radiation intensity has a significant impact on formability and replication accuracy of microstructure; The UV system temperature has a certain influence on microstructure replication accuracy.
\end{abstract}

\section{Introduction}

In recent years with the development of micro-electromechanical technology ${ }^{[1-3]}$, Micro molding manufacturing had been extensively studied and replication accuracy of miniature products had become the research focus ${ }^{[4-6]}$. Meanwhile with the continuous development of mold injection technology ${ }^{[7-8]}$ products was gradually applied in many fields such as sophisticated electronic devices a advanced optics lens optical guided-wave material、nonlinear optical material and gradually replaced the previous inorganic materials, making the manufacturing cost greatly reduced. Microstructure components as advanced technology product were widely needed in many fields, the manufacturing method of microstructure product using mold injection technology had been widely used in research since the $1980 \mathrm{~s}^{[9-11]}$, which found an access to the low cost and mass production ${ }^{[11-12]}$. Curing light was a new manufacturing technology developed for nearly 10 years with characteristics of fast curing speed, low curing temperature and excellent performance..

In the paper light curing molding and traditional mold injection method was combined to research the UV Curing Mold injection of microstructure products on the basis of traditional injection molding process. According to the associated reports ${ }^{[13-16]}$, refractive index of some new UV light curing materials could be more than 1.5, as good as optical PMMA material, possible to be applied in optics products manufacturing. At the same time, UV material with low shrinkage rate was suitable for the micro flow channel mixer. All the merits above lay the foundation for UV light curing to replace thermoplastic material injection molding.

\section{Experiments}

Experimental material. Bifunctional degree of polyurethane acrylates, LE6702, self-help door (heshan) trade co., LTD.;

Three acrylic ester trimethylolpropane, TMPTA, self-help door (heshan) trade co., LTD.; 
Two shrinkage three propylene glycol acrylate, TGPDA, self-help door (heshan) trade co., LTD.; 1 - hydroxy cyclohexyl phenyl ketone, UV184, changsha dextrys polymer technology co., LTD.;

Polypropylene (PP), ST868M, Taiwan LCY.

The main equipments and instruments. UV light curing mold injection test bench, the self-developed;

UVLED ultraviolet light radiation equipment, UPECII - 4 host, UP to 365 head, point light radiation shenzhen especially blue spectrum technology co., LTD.;

High-speed cameras, GVCC04 - B05, American Southern Vision Systems, Inc. (SVSi);

Scanning electron microscope, SP, $250 \mathrm{mk}$, Cambridge company;

All-electric injection molding machine, AE80-32 h, nc equipment co., LTD. Guangzhou.

The experimental project. UV curing mold injection experiment table was as figure 1, 1 - plunger type injection device, 2 - ultraviolet emission lamp holder, 3 - lamp distance adjusting device, 4 press block, 5 - electrical bar, 6 - glass, 7 -thermocouple, 8 - template. Weights were put on the top tray of plunger device to control Injection pressure and the holding pressure; electrical bars were mounted in template adjacent with the runner to maintain UV mixture inside runner at a constant temperature by PID control; briquetting clamped glass to lock the mold; lamp distance adjusting device could move up and down to adjust the distance between lamp holder and the cavity surface. Specimens were as figure 2 .

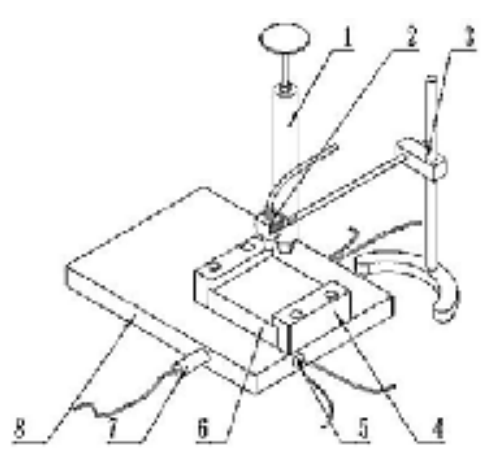

Fig 1. UV curing mold injection experiment table

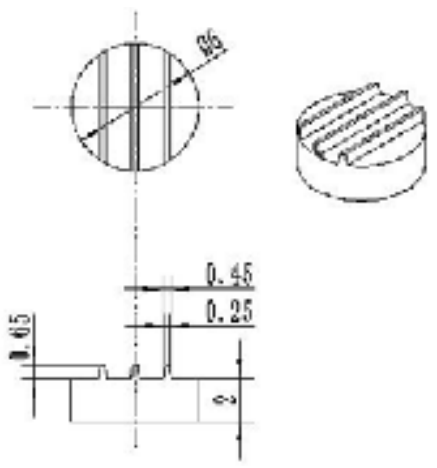

Fig 2. Geometry of the product

In the paper the injection molding technology and UV light curing technology was combined and the UV material temperature and the holding pressure at products were considered as two major factors to value replication accuracy. The quality of the products under different process conditions were measured; The SEM tests were carried on PP injection molding products and UV curing mold injection products and the two microstructure morphologies were compared using image process software

Trapezoidal edge morphology formed under different process conditions was analyzed by microscopic photography and edge height was measured by Image-Pro Plus6.0 to evaluate replication accuracy.

UV curing mold injection experiments. Viscosity of UV resin used in UV curing mold injection experiments under $60{ }^{\circ} \mathrm{C}$ was $8000-15000 \mathrm{cps}$, less than $8000 \mathrm{cps}$ when mixed with some monomer. The experiments showed that cavity could be filled when injection pressure was $4 \mathrm{Mpa}$. According to the experience of mold injection too high holding pressure would cause trimming, therefore holding pressure in these experiments were set at $0.5 \mathrm{Mpa} 、 1 \mathrm{Mpa}$.

In these experiments resin mixture of many UV were used whose forming temperatures were set at $25^{\circ} \mathrm{C} 、 35^{\circ} \mathrm{C}$. Quality method was used to evaluate product forming precision, which was one of indexes measuring replication accuracy. Product quality contained the information of body contraction and microstructure filling. The higher the filling rate, the smaller the volume shrinkage rate (the higher the density), the greater the weight, the products precision was higher. This experiment chose 20 samples under each experimental condition. 
Contrast experiments of mold injection products. With the same micro-structure as object, product forming process was simulated by software Moldex $3 \mathrm{~d}$ to find the proper process parameters. SEM test of light curing forming and PP injection forming were made to compare their cross-sections.

\section{Results and discussion}

The parameter selection of UV light curing injection molding process. UV light curing mold injection was a new forming method. The holding pressure was one of the influences on UV light curing injection molding precision. In experiments the holding pressure were respectively $0.5 \mathrm{Mpa}$ and $1 \mathrm{Mpa}$ at $23,35^{\circ} \mathrm{C}$ and product average quality was measured. When holding pressure was gradually increasing and the material temperature was $25{ }^{\circ} \mathrm{C}$ product average quality percent were respectively $0.325 \%, 0.333 \%$; When holding pressure was gradually increasing and the material temperature was $35{ }^{\circ} \mathrm{C}$ product average quality percent were respectively $0.725 \% 、 0.880 \%$.

The experimental results showed that the quality of products obviously increased with the rise of the holding pressure under the same temperature. It was because when holding pressure increased, the amount of UV material into the cavity increased, then the density of UV material increased.

When holding pressure was $0.5 \mathrm{MPa}$ and UV material temperature was gradually increasing, product average quality percent were respectively $0.780 \%, 1.610 \%$; When holding pressure was $1 \mathrm{MPa}$ and UV material temperature was gradually increasing, product average quality percent were respectively $1.181 \% 、 2.667 \%$; compared with thermoplastic injection molding, light curing injection pressure and curing temperature was low and molding process difficulty was smaller. The advantages above provided certain process basis of light curing injection replacing thermoplastic injection molding.

Mmicro-structure replication accuracy comparison between thermoplastic mold injection and light curing mold injection. In experiments SEM cross-sections of product micro-structure were shot. After proper dealing, the photos of thermoplastic injection molding and light curing injection molding were compared. Figures 3 and 4 showed trapezoidal edge of the UV material and PP material molding.

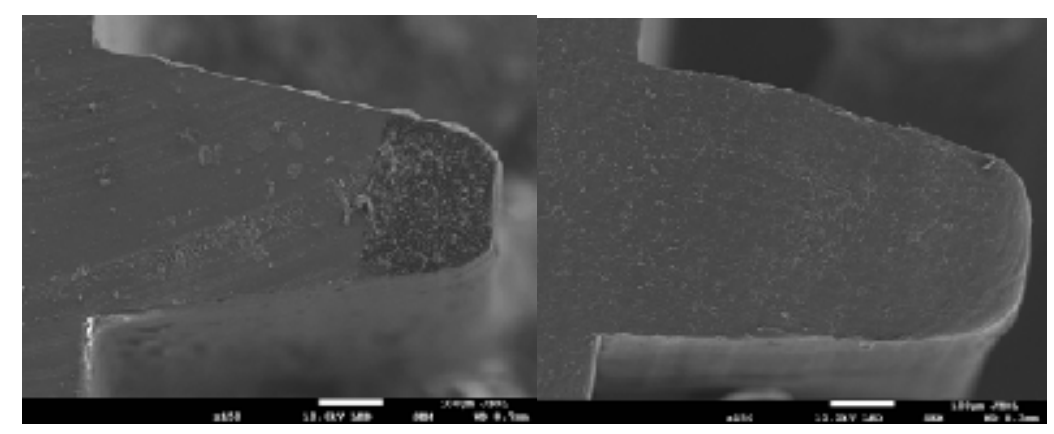

Fig 3.SEM photograph of UV curing injection molding trapezoidal edge Fig 4. SEM photograph of PP injection moldingtrapezoidal edge 


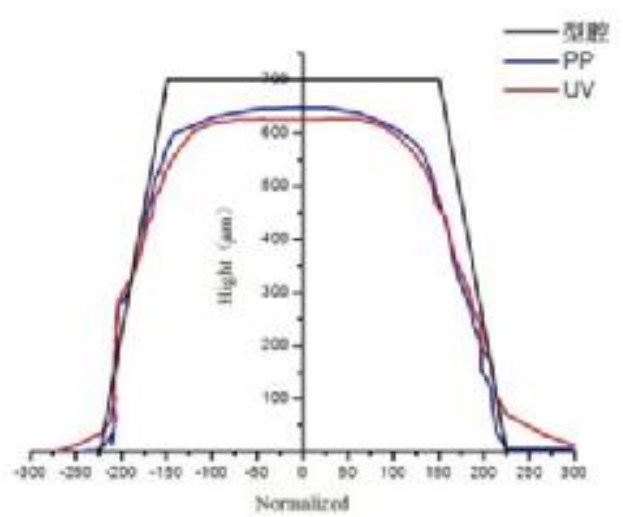

Figure 4 showed trapezoidal edge shape comparison between the UV product and the PP product.

It indicated that trapezoidal edge height of UV product was close to the PP product. Trapezoidal edge height of the PP product was quantitatively $636 \mu \mathrm{m}$ and trapezoidal edge height of UV product was quantitatively $627 \mu \mathrm{m}$.Figure 3,4 showed that trapezoidal edge of UV product keep fine shape. Not considering mechanical performance, thermoplastic injection molding could be instead of UV light curing injection molding.

The effect of UV light energy on UV product replication accuracy.The max radiation intensity of UV-LED point light source used in the experiment was $1000 \mathrm{Wm} / \mathrm{cm} 2$. The radiation energy was increased by the irradiation time. Experimental results indicated that in a certain range, UV product quality improved with the increase of radiant energy. With method of measuring edge height, the effect of process parameters on replication accuracy was studied. Results illustrated when radiation energy increased edge shape was closer to mold core groove in a certain range.

\section{Conclusions}

(1) Holding pressure and material temperature of UV molding process were much lower than thermoplastic injection molding. Thus UV molding process was relatively convenient.

(2) Replication accuracy of UV product was close to the PP product, which illustrated that UV molding process was more promisinig than thermoplastic injection molding.

(3) Higher UV radiant intensity and higher material temperature were beneficial to improve product quality and replication accuracy

\section{References}

[1] Lin, Liwei. Thermal Challenges in MEMS Applications: Phase Change Phenomena and Thermal Bonding Processes. Microelectronics Journal, 34(3), March , $2003: 179$-185.

[2] Osiander, R., Champion, J. L, Darrin, M. A. MEMS in Aerospace Applications-Thermal Control Shutters. Proceedings of SPIE - The International Society for Optical Engineering, 4587, 2001: 7 - 13.

[3] JIANG Bing-yan, SHEN Rui-xia, SHEN Long-jiang, HU Jia-liang, Influence of processing parameters in injection molding on replication fidelity of microstructure parts. Optics and Precision Engineering, 2008, 16(2): 248-256.

[4] YANG Y, GAO F R. Injection molding product weight: online prediction and control based on。 nonlinear principal component regression model[J], Polymer Engineering and Science, 2006, 46(4); 540-548.

[5] Yu Liyong, Koh C q Lee L James, et al. Experimental investigation and numerical simulation of injection molding with micro-features. Polymer Engineering and Science, 2002, 42(5): 871-888. 
[6] Dong Sung Kim, Hyun Sup Lee, Bong-Kee Lee, et al. Replications and Analysis of Microlens Array Fabricated by a Modified LIGA Process. POLYMER ENGINEERING AND SCIENCE, 2006, 46(4):417-425.

[7] Michaeli W, SpennemannA. New plastification concepts for micro injection moulding.Microsystem Technologies,2002, 8(1):55-57.

[8] ZhangPanpan, WangJian, XiePengcheng, YangWeimin.Micro-injection molding technology and differential injection molding technology.CHINA PLASTICS, 2010, 24(6): 13-18.(in Chinese)

[9] SU Y CH, SHAH J, LIN L W. Implementation and analysis of polymeric microstructure replication by micro injection molding[J]. Micromesh. Microeng., 2004, 14(3):415-422.

[10] SHA B, DIMOV S, GRIFFITHS C, et al.. Investigation of micro-injection moulding: factors affecting the replication quality [J]. Journal of Materials Processing Technology, 2007. 183: 284-296.

[11] HECKELE M and SCHOMBURG W K, Review on micro molding of thermoplastic polymers[J]. Micromech Microeng,2004,14(3): 1-14 .

[12] RUPRCHT R, GIETZELT T, MULLER $\mathrm{K}$, et al. Injection molding of microstructrued components from plastics, melts and ceramics[J]. Microsystem Technologies, 2002, 8(4/5): 351-358.

[13] B Y Tay, L Liu N H Loh, et al. Injection molding of 3D microstructures by $\mu$ PIM [J].Microsyst Technol, 2005, 11:210-213.

[14] CUI Z, LV C, YANG B, SHEN J, SU X, YANG B. The research on syntheses and properties of novel epoxy / polymercaptan curing optical resins with high refractive indices. Polymer, 2001, 42: 10095-10100.

[15] CHUJO Y. Organic-inorganic hybrid materials.Current Opinion in Solid\&Materials Science, 1996, 1: 806-811.

[16] LV C, CUI Z, LI Z, YANG B, SHEN J. High refractive index thin films of ZnS / polythiourethanenanocomposites. J. Mater. Chem. 2003, 13: 526-530. [2] W. Strunk Jr., E.B. White, The Elements of Style, third ed., Macmillan, New York, 1979. 